\title{
Comments on Resolution of Nonassociativity in SFT - An Example from Axioms of BCFT -
}

\author{
Matsuo Yutaka \\ Department of Physics, University of Tokyo \\ Hongo 7-3-1, Bunkyo-ku, Tokyo 113-0033, Japan
}

Received on 17 April, 2002

\begin{abstract}
It is known that the associativity of the star product of the open string field theory may be broken in the presence of the nontrivial closed string background. We give an argument that such an anomaly may be resolved by including Chan-Paton factors starting from the axioms of the rational conformal field theory.
\end{abstract}

\section{Introduction}

When Witten has defined the bosonic open string field theory [1], he defined the theory in terms of the noncommutative but associative star product. The noncommutativity is essential feature of the open string field theory since it has two ends and it behaves as a sort of matrix. On the other hand, the associativity is essential to ensure the gauge invariance of Witten's cubic action. These are the key features of the open string field theory when we want to have a correspondence with the noncommutative geometry.

In the explicit calculation of the open string field theory, however, we often meet the nonassociativity of the star product when we want to treat the singular states in the open string Hilbert space. Such a structure appears in the simplified version of the theory, namely treating the open string fields as matrices [2]. The products of three infinite size matrix may not be associative $(A \cdot B) \cdot C \neq A \cdot(B \cdot C)$ since it involves two infinite sums. While each sum may be absolutely convergent, the double sum sometimes becomes only conditionally convergent and it breaks the associativity [3, 4].

This appearance of the associativity anomaly may look rather superficial, namely one may need to be more careful when we define the state and we should rule out the states which breaks the associativity. However, in some situations, the nonassociativity arises from the very nature of the nontrivial closed string background.

We illustrate this phenomena by using two examples.

In the field theory limit, the noncommutative geometry of the string field theory reduces to that of the target space. In the simple situations such as the constant $B$ field in the flat space, the noncommutative geometry is described by the Moyal product which is obviously associative. Even when the $B$ field is not constant, if it satisfies $d B=0$, the target space becomes the Poisson manifold and one may use the Kontsevich's star product to define the associative product. The situation changes drastically when we proceed to define the noncommutative geometry when $d B \neq 0$. In such a situation, Cornalba and Schiappa [5] observed that the naive extension of Kontsevich's star product becomes nonassociative,

$$
f \bullet(g \bullet h)-(f \bullet g) \bullet h \sim(d B)^{i j k} \partial_{i} f \partial_{j} g \partial_{k} h .
$$

At the level of the string field theory, Horowits and Strominger [3] observed that the space-time translation generator may be written in terms of the open string variable as $P_{L} \mathcal{I}$ where $P_{L}$ is the momentum density integrated over half string and $\mathcal{I}$ is the identity operator. Such generators, however, break the associativity because they usually shift the midpoint of the open string. On the other hand, in the conventional definition of the star product, the position of the midpoint is preserved. Strominger[6] later emphasized that such an associativity anomaly is the universal feature of the open string algebra if we want to express the deformation of the closed string background in terms of the open string degree of freedom.

The existence of the associativity anomaly signals that we can not use the conventional description of the noncommutativity. For example, the vector bundle in the commutative geometry is usually translated into the projective module described by the projection operator of the (matrix generalization of the) algebra. In physical terminology, it is described by the noncommutative soliton [7]. However, such an operator does not have any topological meaning once the algebra breaks the associativity.

A possible cure for this problem comes from the observation in the commutative side. When $d B$ be- 
longs to the nontrivial element in the cohomology class $H^{3}(X, \mathbf{Z})$, the gauge transformation generators $\left\{g_{i j}\right\}$ becomes not closed on the intersections of three coordinate neighborhoods $U_{i} \cap U_{j} \cap U_{k}$,

$$
g_{i j} g_{j k} g_{k i}=e^{i \alpha_{i j k}} \neq 1 \text {. }
$$

It shows that the vector bundle becomes twisted and ill-defined.

When $[d B]$ represents a torsion element in $H^{3}(X, \mathbf{Z})$, say $\mathbf{Z}_{n}, e^{i \alpha_{i j k}}$ should satisfy $e^{i n \alpha_{i j k}}=1$. In this special situation, when we adjust the number of D-brane to $n$, the adjoint vector bundle becomes consistent since the phase factor belongs to the center of the gauge group $U(n)$ [8]. By a generalization of this idea, Bouwknegt and Mathai [9] have argued that one may define well-defined adjoint bundle even for generic element of $H^{3}(X, \mathbf{Z})$ when we have the infinite number of D-branes.

A lesson from these phenomena for the noncommutative side is that one may recover the associativity once we include the degree of freedom of D-branes (ChanPaton factor) to modify the originally nonassociative algebra to an associative one. For the generic background, we need the infinite number of D-branes for the modification of the algebra.

The necessity of the infinite number of D-branes suggest that we need a sort of the vacuum string field theory[10]. Namely to express the algebra, we need to employ an infinite dimensional matrix generalization of Witten's string field theory. If all of these Dbranes were physical, we have the infinite energy to create these D-branes. This is not the desired feature of course. By using the vacuum string field theory, one may push most of these D-branes in so called "closed string vacuum". In such a situation, while they contribute to make the algebra associative, they do not cost any energy.

\section{BCFT Axioms and Associa- tivity}

In the axiomatic approach to the Boundary Conformal Field Theory (BCFT) [11], the boundary primary field is characterized by four labels $a, b \in \mathcal{V}, i \in \mathcal{E}$, and $\epsilon=1, \cdots, n_{i}^{a b}$. Here $\mathcal{V}$ represents the possible boundary conditions for the given closed string background. We have two labels $a, b$ because we have two boundaries on the given open string. $\mathcal{E}$ is the set of the chiral primary fields. $n_{i}^{a b}$ represents the number of channels for the primary field $i$ when the boundaries are specified by $a, b$. If we represent Cardy state in terms of Ishibashi state as $\left.|a\rangle=\sum_{i \in \mathcal{E}} \frac{\psi_{i}^{a}}{S_{i 1}}|i\rangle\right\rangle$, we have the relation between them,

$$
n_{i}^{a b}=\sum_{j \in \mathcal{E}} \frac{S_{i j}}{S_{1 j}} \psi_{j}^{a} \psi_{j}^{b *}
$$

which is an analogue of Verlinde formula. By changing $S_{a j}$ (the modular transformation matrices of the character) to $\psi_{j}^{a}$, one may represent CFT for the offdiagonal modular invariant.

We represent the open string Hilbert space with given boundaries $a, b$ as $\mathcal{H}^{a b}$. We write $\Psi_{i \epsilon}^{a b}(z)$ for the boundary primary field and $|i, a, b, \epsilon\rangle$ the corresponding highest weight state. Then $\mathcal{H}^{a b}$ can be represented as

$$
\mathcal{H}^{a b}=\left\{L_{-n_{1}} \cdots L_{-n_{\ell}}|i, a, b, \epsilon\rangle \mid i \in \mathcal{V}, \epsilon=1, \cdots, n_{i}^{a b}\right\}
$$

The star product which we want to define is the mapping,

$$
\star: \mathcal{H}^{a b} \otimes \mathcal{H}^{b c} \rightarrow \mathcal{H}^{a c} .
$$

Equivalently, one may define the star product by using the tri-linear mapping,

$$
V_{3}: \mathcal{H}^{a b} \otimes \mathcal{H}^{b c} \otimes \mathcal{H}^{c a} \rightarrow \mathbf{C} .
$$

In the CFT type definition of the three string vertex [12], the three string vertex operator is defined by the three point function,

$$
\left\langle V_{3}|| A\right\rangle|B\rangle|C\rangle=\left\langle h_{1} \circ A, h_{2} \circ B, h_{3} \circ C\right\rangle .
$$

The conformal transformations $h_{i}$ are defined by

$$
h_{\ell}(z)=e^{\frac{2 \pi i(\ell-1)}{3}}\left(\frac{1+i z}{1-i z}\right)^{2 / 3}, \ell=1,2,3 .
$$

which maps the upper half planes into each piece which divides the unit disk into three. We note that the right hand of (7) depends only on the conformal property of three fields $A, B, C$. Since the conformal weight of the boundary primary fields come only from the label for the primary fields $i$ and not from the boundary indices $\mathcal{V}$, one may calculate the star product from the index $i \in \mathcal{E}$ for the primary field and the operator product expansion for the chiral primary fields,

$\phi_{i}(z) \phi_{j}(w) \sim \sum_{k \in \mathcal{E}} \frac{C_{i j}^{k}}{(z-w)^{h_{i}+h_{j}-h_{k}}} \phi_{k}(w)+($ descendants $)$.

We note that we do not restrict the conformal dimension to be one since we consider the off-shell vertex. The descendants parts of OPE can be deduced from $C_{i j}^{k}$ and the conformal property alone.

Combining these ideas, one arrives at the conclusion that the star product can be in principle defined without using the indices of the boundary. We use the symbol • to denote this definition,

$$
|i, I\rangle \bullet|j, J\rangle=\sum_{k, K} C\left[\begin{array}{lll}
i & j & k \\
I & J & K
\end{array}\right]|k, K\rangle,
$$

where $i, j, k \in \mathcal{E}$ represent the indices for the primary fields and $I, J, K$ are multi-indices for the conformal descendants. 
The particular form of Witten's vertex operator is reflected by the infinite linear relations among the components of the structure constant $C$. It can be derived from the Ward identity for the three string vertex [13]

$$
\left\langle V_{3}\right|\left(L_{v_{n}}^{(1)}+L_{v_{n}}^{(2)}+L_{v_{n}}^{(3)}\right)=0
$$

for the vector fields which are holomorphic in the world sheet of the three string vertex. This relation can be translated into the relations among $C$,

$$
\delta_{n} C\left[\begin{array}{lll}
i & j & k \\
I & J & K
\end{array}\right]=0
$$

These identities are supposed to be sufficient to determine $C\left[\begin{array}{ccc}i & j & k \\ I & J & K\end{array}\right]$ from the OPE coefficient $C_{i j}^{k}$ for the primary fields.

The fact that the chiral primary fields enjoy the nontrivial monodromy implies that the $\bullet$ product which we defined is not associative. In particular, the transformation rule for the chiral block function implies that the structure constant $C$ satisfies,

$$
\begin{aligned}
& \sum_{P} C\left[\begin{array}{lll}
i & j & p \\
I & J & P
\end{array}\right] C\left[\begin{array}{lll}
p & k & l \\
P & K & L
\end{array}\right] \\
& =\sum_{q, Q} F_{p q}\left[\begin{array}{ll}
j & k \\
i & l
\end{array}\right] C\left[\begin{array}{lll}
j & k & q \\
J & K & Q
\end{array}\right] C\left[\begin{array}{lll}
i & q & l \\
I & Q & L
\end{array}\right]
\end{aligned}
$$

The coefficients $F$ is called $6 j$-symbol for the chiral primary fields and satisfies the unitarity condition,

$$
F \bar{F}=1 \text {. }
$$

The consistency condition for $6 j$-symbol was determined as,

$$
F F=F F F
$$

which is called the pentagon identity [14]. Eq.(13) implies the associativity anomaly it implies that

$$
\begin{aligned}
& (|i, I\rangle \bullet|j, J\rangle) \bullet|k, K\rangle=\sum_{p, P} C\left[\begin{array}{lll}
i & j & p \\
I & J & P
\end{array}\right]|p, P\rangle \bullet|k, K\rangle \\
& =\sum_{p, P, l, L} C\left[\begin{array}{lll}
i & j & p \\
I & J & P
\end{array}\right] C\left[\begin{array}{lll}
p & k & l \\
P & K & L
\end{array}\right]|l, L\rangle \\
& |i, I\rangle \bullet(|j, J\rangle \bullet|k, K\rangle)=\sum_{q, Q} C\left[\begin{array}{ccc}
j & k & q \\
J & K & Q
\end{array}\right]|i, I\rangle \bullet|q, Q\rangle \\
& =\sum_{q, Q, l, L} C\left[\begin{array}{lll}
j & k & q \\
J & K & Q
\end{array}\right] C\left[\begin{array}{ccc}
i & q & l \\
I & Q & L
\end{array}\right]|l, L\rangle
\end{aligned}
$$

The existence of the monodromy factor $F$ then suggest the $\bullet$ product is not associative,

$$
(|i, I\rangle \bullet|j, J\rangle) \bullet|k, K\rangle \neq|i, I\rangle \bullet(|j, J\rangle \bullet|k, K\rangle) \text {. }
$$

In the axioms of the boundary conformal field theory, such an anomaly is absorbed in the boundary Chan-Paton index as follows. We include the boundary labels in the definition of the state $|i, I\rangle \rightarrow|i, I, a, b\rangle$. We then define the $\star$ product by using the c-number coefficients $(3 \mathrm{j}$-symbol) as follows,

$$
|i, I, a, b\rangle \star|j, J, b, c\rangle=\sum_{k, K}{ }^{(1)} F_{b p}\left[\begin{array}{cc}
a & c \\
i & j
\end{array}\right] C\left[\begin{array}{ccc}
i & j & k \\
I & J & K
\end{array}\right]|k, K, a, c\rangle
$$

The boundary $3 j$-symbol ${ }^{(1)} F$ is required to satisfy,

$$
\begin{aligned}
& { }^{(1)} F_{b p}\left[\begin{array}{cc}
a & c \\
i & j
\end{array}\right]{ }^{(1)} F_{d p}\left[\begin{array}{cc}
a & c \\
l & k
\end{array}\right] \\
& =\sum_{q} F_{p q}\left[\begin{array}{ll}
i & j \\
k & l
\end{array}\right]{ }^{(1)} F_{a q}\left[\begin{array}{ll}
b & d \\
i & l
\end{array}\right]{ }^{(1)} F_{c q}\left[\begin{array}{ll}
b & d \\
j & k
\end{array}\right]
\end{aligned}
$$


The star product becomes associative because of the unitarity of $6 j$-symbol.

\section{Some comments}

We give some comments and conjectures to be confirmed in the future work in this section.

1. We note that the use of Chan-Paton degree of freedom to describe the associative star product is related to the global anomaly cancellation of the world volume theory on the D-brane [8]. Namely only when we have the appropriate number of Dbranes to cancel anomaly on the world volume. It is interesting to see that this fact is related to the purely algebraic constraint of the associativity anomaly.

2. It is interesting to study this anomaly cancellation mechanism can be applied to the HorowitzStrominger anomaly [3]. One possibility to answer this problem may be the following. Horowitz-Strominger anomaly arises when we need to describe the space-time reparametrization (namely the gauge degree of freedom of the closed string). In CFT language, it amounts to the marginal deformation of the closed string background. Such a deformation of the theory induces the redefinition of the primary fields and at the same time their OPE relations. In the conventional operator formalism, there is no explicit dependence of Chan-Paton factors and one arrives at anomaly because of nontrivial $6-j$ symbol in this background.

3. As we commented in the introduction, the framework of the vacuum string field theory (VSFT) is necessary to make our discussion consistent. The VSFT conjecture may be summarized as follows. When the open string tachyon is condensed, we are supposed to arrive at the "closed string" vacuum. The description of such a state is given by changing the conventional BRST charge $Q$ to the background independent one $\mathcal{Q}$ which is described only in terms of the ghost field. This operator $\mathcal{Q}$ should not have physical spectrum since in the "closed string" vacuum there is no D-branes. When D-brane is excited from the vacuum, the string field is shifted,

$$
\Psi \rightarrow \Psi_{0}+\delta \Psi
$$

and expansion around this new vacuum gives a new BRST charge,

$$
\left.Q=e^{K}\left(\mathcal{Q}+[\ominus,]_{\star}\right)\right\rceil^{-\mathcal{K}}
$$

with an appropriate "unitary transformation" $K$. $\Psi_{0}$ is described by the projection operator of $\star$ product and $Q$ should have nontrivial physical states which lives on the created D-brane. Due to the nonzero shift $\Psi_{0}$, the vacuum energy is shifted by the value of the action $S\left(\Psi_{0}\right)$ which can be interpreted to give the tension of the D-brane.

In our discussion, when the closed string background is generic, the monodromy matrix $F$ can be canceled only when we introduce the infinite number of D-branes. If all of these D-branes should be physical, we suffer from the infinite contribution of the D-brane tension. This is inconsistent. So if we only have the open string field theory of physical D-branes, we can not define the open string degree of freedom at all. However, if we deform the closed string background to more trivial one such as flat space, the existence of the finite number of the D-branes becomes possible and we need the open string field theory which describe them. In this sense, the behavior of the open string field theory heavily depends on the closed string background which can be continuously deformed. It implies that we have a discontinuity of the description of the open string degree of freedom in the moduli space of the closed string background.

If we use the VSFT scenario, however, one may keep the infinite number of D-branes hidden in the background without consuming energy. When background changes, the open string algebra changes continuously and at some specific points, one may construct the projection operator of $\star$ product. Therefore in this framework, there is no discontinuity of the description of the open string. The discontinuity depending on the background comes from the consistency of the construction of the projection operator, which looks more natural to work with. One possible strategy to define VSFT with Chan-Paton factor is discussed in [15].

Acknowledgement: The author would like to thank Prof. Berkovits for inviting him to Brazil, many discussions and a warm hospitality during his stay. He is also obliged to the organizer for giving the oppotunity to present his result in "XXII Encontro Nacional de Fisica de Particulas e Campos".

\section{References}

[1] E. Witten, Nucl. Phys. B268, 253 (1986).

[2] D. J. Gross and W. Taylor, JHEP 0108, 009 (2001) [arXiv:hep-th/0105059].

D. J. Gross and W. Taylor, JHEP 0108, 010 (2001) [arXiv:hep-th/0106036]. 
T. Kawano and K. Okuyama, JHEP 0106, 061 (2001) [arXiv:hep-th/0105129].

[3] G. T. Horowitz and A. Strominger, Phys. Lett. B 185, 45 (1987).

[4] I. Bars and Y. Matsuo, "Associativity Anomaly In String Field Theory", hep-th/0202030.

[5] L. Cornalba and R. Schiappa, Commun. Math. Phys. 225, 33 (2002) [arXiv:hep-th/0101219].

[6] A. Strominger, Nucl. Phys. B 294, 93 (1987).

[7] R. Gopakumar, S. Minwalla, and A. Strominger, JHEP 0005, 020 (2000) [arXiv:hep-th/0003160].

[8] D. S. Freed and E. Witten, arXiv:hep-th/9907189.

[9] P. Bouwknegt and V. Mathai, JHEP 0003, 007 (2000) [arXiv:hep-th/0002023].

[10] See for example, L. Rastelli, A. Sen and B. Zwiebach, JHEP 0111, 045 (2001) [arXiv:hep-th/0105168];
D. Gaiotto, L. Rastelli, A. Sen, and B. Zwiebach, arXiv:hep-th/0111129.

[11] R. E. Behrend, P. A. Pearce, V. B. Petkova, and J. B. Zuber, Nucl. Phys. B 570, 525 (2000); [Nucl. Phys. B 579, 707 (2000)] [arXiv:hep-th/9908036]; Y. B. Petkova and J. B. Zuber, Nucl. Phys. B 603, 449 (2001) [arXiv:hep-th/0101151].

[12] A. LeClair, M. E. Peskin, and C. R. Preitschopf, Nucl. Phys. B 317, 411 (1989); A. LeClair, M. E. Peskin, and C. R. Preitschopf, Nucl. Phys. B 317, 464 (1989).

[13] L. Rastelli and B. Zwiebach, JHEP 0109, 038 (2001) [arXiv:hep-th/0006240].

[14] G. W. Moore and N. Seiberg, Commun. Math. Phys. 123, 177 (1989).

[15] Y. Matsuo, Mod. Phys. Lett. A 16, 1811 (2001) [arXiv:hep-th/0107007]. 